\title{
Endoscopic Papillectomy for Adenocarcinoma at the Duodenal Papilla - Will It Still Open a Pandora's Box?
}

\author{
Masaki Kuwatani $^{1,2}$ and Naoya Sakamoto ${ }^{2}$ \\ Key words: endoscopic papillectomy, endoscopic resection, major papilla; minor papilla, adenocarcinoma, \\ adenoma
}

(Intern Med 60: 2523-2524, 2021)

(DOI: 10.2169/internalmedicine.7125-21)

Since the first instance of endoscopic papillectomy for a lesion at the duodenal papilla (the ampulla of Vater) was reported by Suzuki et al. in 1983 (1), endoscopists have used this approach to manage various lesions, such as adenoma mainly but rarely neuroendocrine tumor, lymphoma, ganglyocytic paraganglioma, and adenocarcinoma at the major duodenal papilla (MaDP) and minor duodenal papilla (MiDP). However, endoscopic papillectomy remains challenging procedure for experts to perform because it has potential risks of non-curative resection and severe adverse events (generally about 5-10\%) (2), such as major bleeding, perforation and pancreatitis, especially in the area of the MiDP, where there is no evidence for the curability and safety of endoscopic papillectomy. At the MaDP, successful papillectomy rates for adenomas range from $46 \%$ to $92 \%$, whereas recurrence rates have been reported to be as high as $33 \%$. To solve these issues, we must establish a diagnostic system with high accuracy and a treatment procedure with high safety and curability rates, although there will still be a long way to go to achieve them.

With this background, Sato et al (3). reported successful endoscopic mucosal resection (EMR) of adenocarcinoma at the MiDP and highlighted the associated issues. As Sato et al. noted, since there have been only three cases of adenocarcinoma at the MiDP curatively treated by EMR, it is difficult to adequately address the relevant issues using cases of adenocarcinoma at the MiDP alone. Therefore, the issues with and feasibility of EMR of adenocarcinoma at the duodenal papilla must be discussed according to the outcomes at the MaDP as well as those at the MiDP, including adenomas and other neoplasms at both the MaDP and MiDP.

Although endoscopic papillectomy for cancer at the MaDP, which has been suggested to be a curative treatment in early stages of adenocarcinoma (Tis and T1), has indi- cated good results in the literature (2), those findings were limited to case reports, which are likely to suffer from publication bias. Nevertheless, the abovementioned findings concerning endoscopic papillectomy at the MaDP expand the possibility of its application to adenomas and adenocarcinomas at the MiDP with similar criteria: 1) adenoma with high-grade dysplasia/non-invasive (not beyond the submucosal layer of the duodenum) adenocarcinoma component and 2) no (or $<1 \mathrm{~cm}$ ) extension into the pancreatic duct (2).

For the accurate pretreatment diagnosis of lesions at the MaDP or MiDP, we perform a forceps biopsy under endoscopy and use imaging modalities, such as multi-detector row computed tomography, magnetic resonance imaging with high resolution, intraductal ultrasonography (IDUS) under endoscopic retrograde cholangiopancreatographyguidance and endoscopic ultrasonography (EUS) with/without elastography. A forceps biopsy reflects only the focal and superficial pathological condition of a lesion and occasionally causes pancreatitis due to edema after the biopsy at the MaDP or MiDP in cases with pancreas divisum. Therefore, forceps biopsies at the duodenal papilla should be cautiously performed, even when necessary for differentiating papillitis, adenoma, and adenocarcinoma.

Among imaging modalities, IDUS and EUS have played a central role in the diagnosis of the vertical and horizontal margins of lesions at the MaDP and MiDP, focusing on the sphincter of Oddi, muscular layer of the duodenum, and layer structure of the bile/pancreatic duct (2). The accuracies of IDUS and EUS in T staging of cancer at the MaDP were reported to be $78-93 \%$ and $62-63 \%$, respectively (4). The difference in the accuracies can be attributed to the distance from the target and frequency of each echo probe. However, it is occasionally impossible to insert an IDUS probe via the MiDP in patients with a small orifice, which is congenitally

${ }^{1}$ Division of Endoscopy, Hokkaido University Hospital, Japan and ${ }^{2}$ Department of Gastroenterology and Hepatology, Hokkaido University Hospital, Japan

Received: January 19, 2021; Accepted: January 28, 2021; Advance Publication by J-STAGE: March 15, 2021

Correspondence to Dr. Masaki Kuwatani, mkuwatan@med.hokudai.ac.jp 
occluded in $\leq 50 \%$ of cases (5). Thus, IDUS and EUS should complementarily be used for a local diagnosis at the MiDP.

Regarding the anatomy of the MiDP, whether or not the MiDP has a sphincter, which would be smoothly connected to the proper muscular layer of the duodenum, is controversial, even from a pathological viewpoint (5). Regardless, we should concentrate on whether or not a lesion at the MiDP infiltrates the proper muscular layer (possibly including a sphincter) and whether or not it intraductally extends into the pancreatic duct, with IDUS and EUS used for the appropriate selection of candidates for endoscopic papillectomy.

The basic technique for endoscopic papillectomy-snare resection-has been unchanged for approximately 40 years. The pancreas is very susceptible to physical and chemical stimulation, and circulation and edema of the papilla can lead to pancreatitis. Submucosal injection is effective for preventing gastrointestinal perforation, ensuring a negative margin around a lesion, and confirming a deep lesion with a non-lifting sign. However, submucosal injection at the duodenal papilla is controversial. Fluids injected into the submucosa of the MaDP have included saline solution, epinephrine, and viscous materials, such as hydroxypropyl methylcellulose, and similar injections have been performed at the MiDP in most cases with adenoma and three cases with adenocarcinoma treated by endoscopic papillectomy (3). As Sato et al. discussed, submucosal injection at the MiDP without pancreas divisum is reasonable and beneficial for the prevention of remnant tumor and duodenal perforation because of the sparse structure of the MiDP lined with discontinuous pancreatic tissues to the proper pancreas in $60 \%$ cases (5), although such submucosal injection may occasionally make snaring the papilla difficult. Further investigations are therefore required.

Also debatable is whether or not a prophylactic pancreatic stent should be placed after endoscopic papillectomy. A retrospective study of 82 patients showed that there was no need for routine pancreatic stent placement, while the only randomized controlled trial performed to date, with 19 patients, reported a reduction in the rate of post-ERCP pancreatitis by placing a pancreatic stent after endoscopic papillectomy at the MaDP (2). Taken together, the American Society of Gastrointestinal Endoscopy Committee recommends prophylactic pancreatic duct stent placement and rectal indomethacin during papillectomy at the MaDP to reduce the risk of postprocedural pancreatitis (2). However, the situation is different for papillectomy at the MiDP, as the outflow of pancreatic juice is usually-without pancreas divisummaintained via the MaDP even if the MiDP is occluded. Of note, the difference in the efficacy between pancreatic stenting and endoscopic nasopancreatic drainage that Sato et al. performed has yet to be determined.

There have been several recent reports regarding novel techniques for endoscopic papillectomy at the MaDP based on snare resection. One involves underwater EMR without submucosal injection for resection of ampullary adenomas, in which water submersion causes the adenoma to float, facilitating ensnarement (2). However, a case with iatrogenic duodenal perforation after underwater EMR of the MaDP has been reported. Another novel technique of hybrid endoscopic papillectomy involves the effective combination of snare resection with endoscopic submucosal dissection as a proof-of-concept study with eight patients (6). In addition, it has also been reported that cold polypectomy, which is a new technique without submucosal injection and electric cautery for colonoscopic polypectomy, at the MiDP can cause severe pancreatitis (7). However, in that case, the authors misidentified the MiDP as duodenal adenoma not located at the MiDP. Furthermore, they found that the case had incomplete pancreas divisum by magnetic resonance cholangiopancreatography after cold polypectomy. Thus, this previous incident does not directly deny the feasibility of cold polypectomy at the MiDP, although this procedure should be considered cautiously and carefully.

Although there are still some concerns and issues in performing endoscopic papillectomy at both the MaDP and MiDP, they may be resolved through repeated discussion and hard work to develop a novel technical evolution. Whether or not endoscopic papillectomy for adenocarcinoma at the duodenal papilla will open a Pandora's box also depends on them.

The authors state that they have no Conflict of Interest (COI).

\section{References}

1. Suzuki K, Kantou U, Murakami Y. Two cases with ampullary cancer who underwent endoscopic excision. Prog Dig Endosc 23: 236-239, 1983.

2. ASGE Standards of Practice Committee; Chathadi KV, Khashab MA, Acosta RD, et al. The role of endoscopy in ampullary and duodenal adenomas. Gastrointest Endosc 82: 773-781, 2015.

3. Sato T, Sagami R, Nishikiori H, et al. Endoscopic mucosal resection of adenocarcinoma at the minor duodenal papilla: a case report and suggestions for the optimal treatment strategy. Intern Med 60: 2593-2599, 2021.

4. Ito K, Fujita N, Noda Y, et al. Preoperative evaluation of ampullary neoplasm with EUS and transpapillary intraductal US: a prospective and histopathologically controlled study. Gastrointest Endosc 66: 740-747, 2007.

5. Suda K. Histopathology of the minor duodenal papilla. Dig Surg 27: 137-139, 2010.

6. Takahara N, Tsuji Y, Nakai Y, et al. A novel technique of endoscopic papillectomy with hybrid endoscopic submucosal dissection for ampullary tumors: a proof-of-concept study (with video). J Clin Med 9: 2671, 2020.

7. Akimoto T, Kato M, Yahagi N. Severe acute pancreatitis following cold polypectomy of the minor duodenal papilla in a case with pancreas divisum. Dig Endosc 32: 151, 2020.

The Internal Medicine is an Open Access journal distributed under the Creative Commons Attribution-NonCommercial-NoDerivatives 4.0 International License. To view the details of this license, please visit (https://creativecommons.org/licenses/ by-nc-nd/4.0/).

(C) 2021 The Japanese Society of Internal Medicine

Intern Med 60: 2523-2524, 2021 